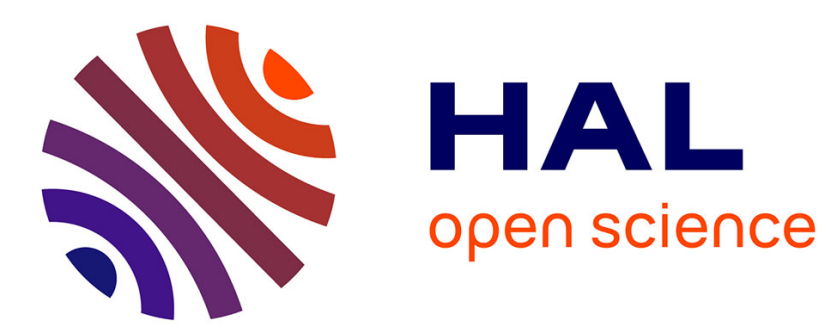

\title{
Check and validate reed solomon block turbo codes in shallow underwater acoustic communication
} Joël Trubuil, André Goalic, Nicolas Beuzeulin, Christophe Laot

\section{To cite this version:}

Joël Trubuil, André Goalic, Nicolas Beuzeulin, Christophe Laot. Check and validate reed solomon block turbo codes in shallow underwater acoustic communication. Oceans '10, May 2010, Sydney, Australia. hal-00523108

\section{HAL Id: hal-00523108 https://hal.science/hal-00523108}

Submitted on 10 Jun 2021

HAL is a multi-disciplinary open access archive for the deposit and dissemination of scientific research documents, whether they are published or not. The documents may come from teaching and research institutions in France or abroad, or from public or private research centers.
L'archive ouverte pluridisciplinaire HAL, est destinée au dépôt et à la diffusion de documents scientifiques de niveau recherche, publiés ou non, émanant des établissements d'enseignement et de recherche français ou étrangers, des laboratoires publics ou privés. 


\title{
Check and validate Reed Solomon Block Turbo Codes in Shallow Underwater Acoustic Communication
}

\author{
Joël Trubuil" ${ }^{*}$ André Goalic ${ }^{*}$, Nicolas Beuzelin ${ }^{* *}$ and Christophe Laot ${ }^{*}$ \\ ${ }^{*}$ TELECOM Bretagne, BP 83818, 29238 Brest Cedex 3, France \\ ${ }^{* *}$ G.E.S.M.A. BP 42, 29240 Naval Brest, France
}

\begin{abstract}
In collaboration with TELECOM Bretagne, GESMA (Groupe d'Etudes Sous-Marines de l'Atlantique) has recently developed a platform for data transmission via broadband underwater acoustic link. The acoustic link must be robust and have a bit rate high enough to transmit different kinds of information. The platform, called TRIDENT presents interesting prospects for applications involving transmission of data in text, image, and also speech. Recently this demonstrator has been modified to extend with existing treatments, the tools able to sample, compress the speech signal, before the modulation and the transmission step. The acoustic link uses channel coding options based on convolutional codes (CC) and block codes Reed Solomon (RS). The need was for the purpose of evaluation capacity and performance of an error correcting code belonging to the Turbo Codes family. Thus a new code correction using Reed Solomon Block Turbo Codes (RS BTC) has been incorporated into the platform. The data transmitted are interleaved and frame recovery is performed in reception. An evaluation of these contributions has been conducted in Brest bay.
\end{abstract}

Keywords: Underwater acoustic communication, BER (Bit Error Rate), Synchronization, Reed Solomon Block Turbo Codes (RS BTC)

\section{INTRODUCTION}

A few years ago, GESMA (Groupe d'Etudes Sous-Marines de l'Atlantique), in collaboration with TELECOM Bretagne, launched the TRIDENT (TRansmission d'Images et de Données EN Temps réel) project. The first objectives were to equip autonomous underwater vehicles (AUVs) with a wireless communication system. This system was designed for continuous data transmission and is able to transmit data such as images, speech and text.

The TRIDENT system [1] can use four carrier frequencies $(11.2,17.5,20.0$ and $34 \mathrm{kHz})$. The bit rate under consideration range from 2.8 to $23.3 \mathrm{kbps}$ with a QPSK modulation (Quadrature Phase Shift Keying). Multipath propagation, noise and Doppler effect are responsible of most of the distortion on underwater acoustic communication. These physical impacts bring to a time dispersion and a variability to the received signal. One can note that carrier frequency and available bandwidth are much lower than those existing in other communication channels. A blind spatio-temporal equalizer [2] is used to reduce these various perturbations.

GESMA also aims to increase the link's reliability. The main objective is to decrease the BER from $10^{-2}$ to $10^{-4}$. In order to do so, a channel coding was integrated to the system. Two kinds of error correcting schemes has been tested including Convolutional Codes (CC) and Reed Solomon (RS) block code[3][4]. These two tested channel coding schemes have a low code rate $(0.5$ for the $\mathrm{CC}$ and 0.7 for the RS) and do not bring enough correction.

Also, to improve the symbol correction and obtain a higher code rate, Reed Solomon Block Turbo Codes (RS BTC) was tested. The iterative decoding process based on the Chase algorithm is a soft version (4 iterations). The soft decoding process uses also Berlekamp and Chien algorithms to correct received symbols. Three different synchronization words are wisely included in the coded frame to synchronize the interleaver and recover the information frame at the receiver. A differential coding/decoding with soft decoding is used to solve the phase ambiguity. The use of differential coding decreases performance in doubling the bit error rate. However, the use of differential coding is necessary to allow the detection of possible phase jumps and symbol errors introduced.

This paper provides an overview of the high data rate acoustic link. Firstly, we present the TRIDENT platform and the extension with the RS Block Turbo Codes. We describe the strategy for synchronizing the interleaver and the channel decoder, and solving the phase ambiguities. Then, we describe the sea trials and show the first acoustic 
communication results in the 2.8 to $14 \mathrm{kbps}$ range, applied both to images and low bit rate speech $(2400 \mathrm{bps})$ transmission. Sea trials took place in November 2009 in the Bay of Brest. The emitter and the receiver were in stationary state, in order to validate the algorithms.

\section{ITERATIVE DECODING OF REED SOLOMON BLOCK TURBO CODES}

The underwater acoustic channel is characterized mainly by strong fading, multipath, noise and Doppler effect. As part of the wireless communication system TRIDENT, the preferred technique for transmitting information is to use a Quadrature Phase-Shift Keying (QPSK) modulation, associated in reception, with a blind spatio-temporal equalization. This equalizer uses input signals sampled on several sensors (4 in this version) only coming from a single emission source. This space diversity improves the Signal to Noise Ratio (SNR) compared to the mono-sensor version. This equalizer is able to run according a convergence or starting mode and a tracking mode, which are differentiated both on the structure and the algorithm. The interest of this adaptivity lies in the possibility of switching from one structure to another according to the channel severity. Moreover, it is not necessary to use a preamble or other training sequence, only the user data are transmitted. This approach seems particularly appropriate for implementation simplicity, interoperability and better spectral efficiency. By using a higher code rate, the user bit rate is higher for a transmission bit rate fixed. To improve the underwater acoustic link performances and obtain a higher code rate, Reed Solomon Block Turbo Codes (RS BTC) was tested.

The concept of product code introduced by P. Elias in 1954 is a single and efficient method to construct powerful codes. Product codes are obtained by means of serial concatenation of two (or more) linear block codes, $e_{1}$ having parameters $\left(n_{1}, k_{1}\right.$, $\left.\delta_{1}\right)$ and $e_{2}$ having parameters $\left(n_{2}, k_{2}, \delta_{2}\right) . n_{i}, k_{i}$ and $\delta_{i}(i=1,2)$ stand respectively for code length, code dimension and Minimum Hamming Distance (MHD) of each code. The parameters of the constructed product code $P$ are given by $n=n_{1}$ $\times n_{2}, k=k_{1} \times k_{2}, \delta=\delta_{1} \times \delta_{2}$. The code rate of $P$ is $R=R_{1} \times$ $R_{2}$ where $R_{i}$ is the code rate of its component codes. It is shown [5] that all $n_{1}$ rows of the matrix are code words of $e_{1}$ just as all $n_{2}$ columns are code words of $e_{2}$ by construction.

RS codes are a subclass of non-binary $\mathrm{BCH}$ codes. $\mathrm{RS}(n, k$, $\delta$ ) is a Maximum Distance Separable (MDS) code which has the minimal code length for a given code rate and error correcting capability. RS product codes can constructed based on either Qary symbol concatenation or bit concatenation. In order to get smaller block size, Q-ary symbol based RS product code is used here. It is constructed from $\left(k_{1} \times k_{2}\right)$ Q-ary $\left(Q=2^{q}\right)$ information symbols, which means $\left(k_{1} \times k_{2} \times \quad q\right)$ data bits. The $q$ binary elements corresponding to a Q-ary symbol are at the intersection of a row and a column. Serial concatenation is realized by coding Q-ary symbols along the rows and columns successively. Our RS product code $P$ is constructed by using two identical component codes $e_{1}=e_{2}=\operatorname{RS}(n, k, \delta)$ and is shown in Figure 1.

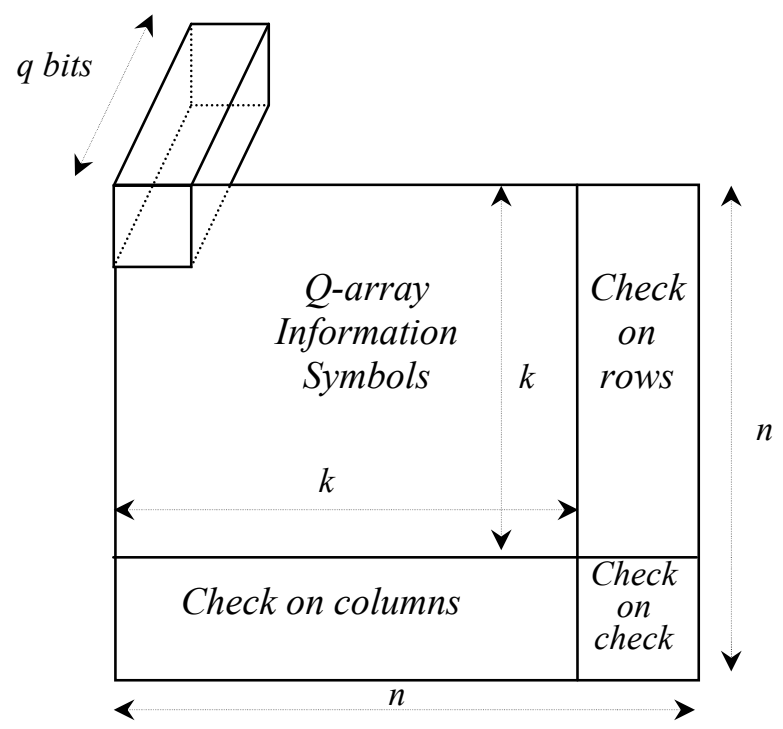

Figure 1 : RS product codes with Q-ary symbol concatenation

By using a Galois Field (GF) generator polynomial [6], the above RS product code can be represented by a matrix of $\mathrm{n}$ rows and $(n \times q)$ columns after the Q-ary to binary conversion.

The transmission with QPSK modulation over Additive White Gaussian Noise (AWGN) channel can be regarded as the superposition of two independent Binary Phase-Shift Keying (BPSK) modulations applied respectively to the inphase and orthogonal carrier. Each bit of the RS product code is associated with one binary value according to the mapping rule $(0 \rightarrow-1,1 \rightarrow+1)$. At the channel output, the bit level Log-Likelihood Ratio (LLR) is computed and fed to the following turbo decoder.

The turbo decoder is made up of several cascaded SoftInput-Soft-Output (SISO) decoder. Each SISO decoder is used for decoding rows and columns. A SISO decoder can be divided into Soft-Input-Hard-Output (SIHO) decoder and a reliability estimation of each decoded bit at the output of the SIHO decoder. The Chase's algoritm [7] is extended to construct the SIHO decoder for RS $(n, k, \delta)$ over GF(Q). Let the vector $R=\left(r_{1}, r_{2}, \ldots, r_{i}, \ldots, r_{q n}\right)$ be the normalized bit level LLR sequence after soft demodulation for one received word and $Y=\left(y_{1}, y_{2}, \ldots, y_{i}, \ldots, y_{q n}\right)$ be the hard 
decision estimation of the transmitted sequence following the equation 1.

$$
y_{i}=0.5 \times\left[\operatorname{sign}\left(r_{i}\right)+1\right] \text { for } 1 \leq i \leq(q \times n)
$$

Firstly the $s$ least reliable positions corresponding to the smallest absolute values in vector $R$ are determined. Test patterns of length $q n$ of weight " 0 " to $s$ with the " 1 " restricted to the least reliable positions are added to the sequence $Y$ to obtain new binary sequences. A Q-ary sequence $H=\left(h_{1}, h_{2}, \ldots, h_{i}, \ldots\right.$, $\left.h_{n}\right)$ is then obtained based on new binary sequence. The algebraic RS decoder processes these Q-ary sequences and produces the Maximum-Likelihood (ML) RS codewords. These ML codewords are then represented by binary sequence of length $q n$ after the Q-ary to binary decomposition and noted $C^{i}$. The Chase algorithm computes the Euclidian Distance (ED) to the input $R$ and selects codeword $D$ at minimum ED from $R$. For each bit $d_{j}(1 \leq j \leq q n)$ of $D$, we search for the concurrent code word $C$ at minimum ED from $R$ such that $c_{j} \neq d_{j}$ at positions $j$. If such codeword is available, the soft output for bit $d_{j}$ is given by (2) [8].

$$
r_{j}^{\prime}=\left(\frac{M^{C}-M^{D}}{4}\right) \times d_{j}
$$

where $M^{D}$ is ED between $D$ and $R$ and $M^{C}$ is ED between $R$ and $C$. Else we use the predefined value $\beta$ and the following relation (3).

$$
r_{j}^{\prime}=r_{j}+\beta d_{j}
$$

The extrinsic information $W$ which is the difference between soft output and soft input for each component is computed and sent to the next SISO decoder. Input to the next SISO decoder is given by $R+\alpha W$ where $\alpha$ is used to reduce the influence of extrinsic information in the first iteration.

The complexity of this SISO decoder depends on parameter $s$. Given that the soft decoding criterion is to find the RS binary codeword of length $q n$ at minimum ED from observation vector $R$, then $s$ is given by $\delta_{b} / 2$ where $\delta_{b}$ is the minimum Hamming distance of RS binary codewords. In the case of single-error correcting codes, $\delta_{b}=\delta=3$ and thus s $=1$ is sufficient for SIHO decoding.

\section{FRAME SYNCHRONIZATION AND DIFFERENTIAL CODING}

Information transmitted is generally framed. In reception, synchronization words are conventionally used to synchronize both the source and channel decoders. Interleaver need another synchronization. Several level of synchronization word lowers the whole correcting rate but makes it more flexible design of different transmission system stage.

In the TRIDENT system, with the RS BTC channel coding, three different synchronization words $S_{i}(i \in\{1,2,3\})$ are included to retrieve the frame, at the receiver. $S_{1}$ (respectively $S_{2}$ or $S_{3}$ ) is used to detect the frame start (respectively middle or end frame). The selected sequences are quasi-orthogonal with good correlation properties. Figure 2 show the autocorrelation and cross-correlation sequences. From these graphs, we can set a threshold of the frame detection. Synchronization words can be include in or out the Q-array information symbols. They are included before the interleaver. This will make them less sensitive to burst errors. The emitted coded frame, with $S_{i}$ in (respectively out) the frame, is 4805 bits in length (respectively 5084), thus the whole correcting rate is $R=$ 0.82 .
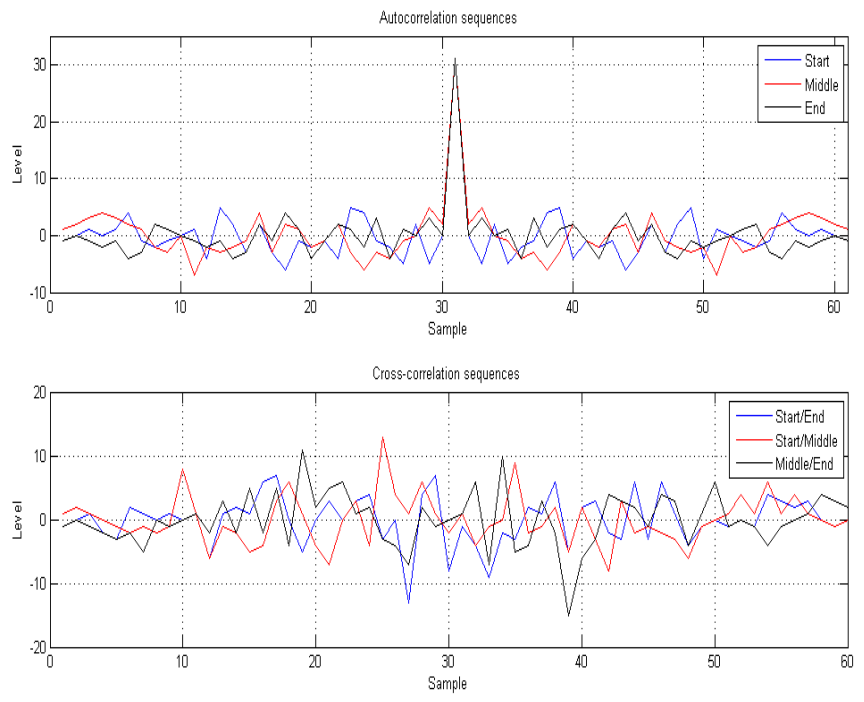

Figure 2. Autocorrelation and cross-correlation sequences

As we intrinsically have a synchronization words, it is likely that the differential coding, used to correct the phase ambiguity, becomes useless. Let us recall that differential coding leads to a $3 \mathrm{~dB}$ loss in terms of coding gain. Having a look to the frame length, the differential coding is required. Indeed, a phase jump at the frame start can generate too many errors and the expectation of a new detection is too long. Two kinds of differential decoding (DC) have been considered.

First possibility of DC use a hard decoding process (noted DC Hard). Hard decisions data decided are used to 
detect the phase ambiguity and correct samples phase for the soft channel decoding.

Second possibility of DC use a soft differential decoding (noted DC LLR ). The Log-Likelihood Ratio is used to solve the phase ambiguity following equation (4).

$$
\text { and } \begin{aligned}
\Lambda\left(\alpha_{\mathrm{n}}\right) & =\ln \frac{\operatorname{Pr}\left\{\alpha_{\mathrm{n}}=1 / \mathrm{y}_{\mathrm{n}}\right\}}{\operatorname{Pr}\left\{\alpha_{\mathrm{n}}=0 / \mathrm{y}_{\mathrm{n}}\right\}} \\
& \left(\beta_{\mathrm{n}}\right)=\ln \frac{\operatorname{Pr}\left\{\beta_{\mathrm{n}}=1 / \mathrm{y}_{\mathrm{n}}\right\}}{\operatorname{Pr}\left\{\beta_{\mathrm{n}}=0 / \mathrm{y}_{\mathrm{n}}\right\}}
\end{aligned}
$$

In equation (4), $y_{n}$ represents the output equalizer sample. $\alpha_{n}$ and $\beta_{n}$ correspond respectively to the real and imaginary parts of the decided data. $\Lambda\left(\alpha_{n}\right)$ and $\Lambda\left(\beta_{n}\right)$ represent respectively the real and imaginary parts of the output equalizer sample, corrected in phase.

\section{RESULTS}

To improve the performance of the TRIDENT acoustic link, Reed Solomon Block Turbo Codes are under consideration. Channel coding goal is to lower the bit error rate from $10^{-2}$ to $10^{-4}$ and improve images or speech synthesis quality. After synchronization and equalization efficiently realized, channel coding may add extra performance.

\section{A. Simulations}

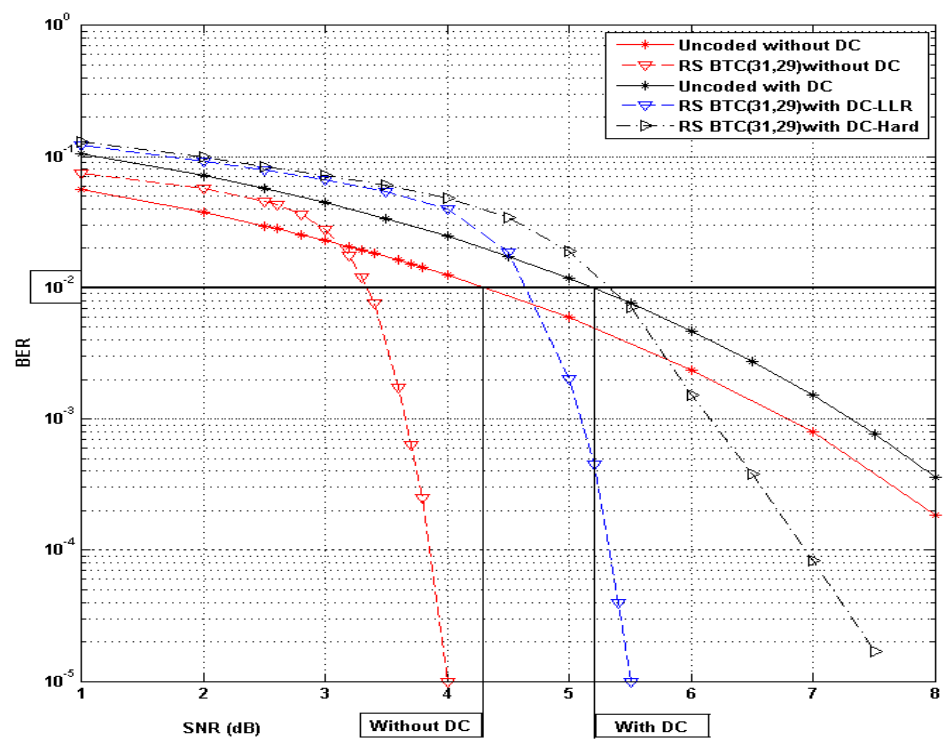

Figure 3. RS BTC with and without differential decoding on Gaussian Channel

The first results presented are obtained by simulations in presence of Additive White Gaussian Noise. Figure 3 presents performance obtained with Reed Solomon Block Turbo Codes.
It clearly appears that differential coding (DC) decreases performance by doubling the bit error rate. We take the BER at the output of the channel as a reference for the 3 options, without DC and with DC Hard or LLR. When using DC both for hard and soft decoding, we can not lower the BER from $10^{-2}$ to $10^{-4}$. Let us recall that in the case of phase ambiguity, the decoding process may not work correctly. The use of synchronization words $S_{i}$ is able to solve the phase ambiguities. Only without DC, we obtain the performance wished. Several phase jumps can appear during the frame transmission. Thus, it is better to use differential encoding with LLR decoding.

Figure 4 presents an image transmission on AWGN channel with a SNR at $5.5 \mathrm{~dB}$. The BER at the channel decoder input is $0.810^{-2}$ and $1.710^{-5}$ at the output. Performance are obtained with a differential coding using LLR. We can see that with one iteration of decoding channel, residual errors can not reconstruct the image correctly. After 4 iterations, the few remaining errors do not degrade the image quality.

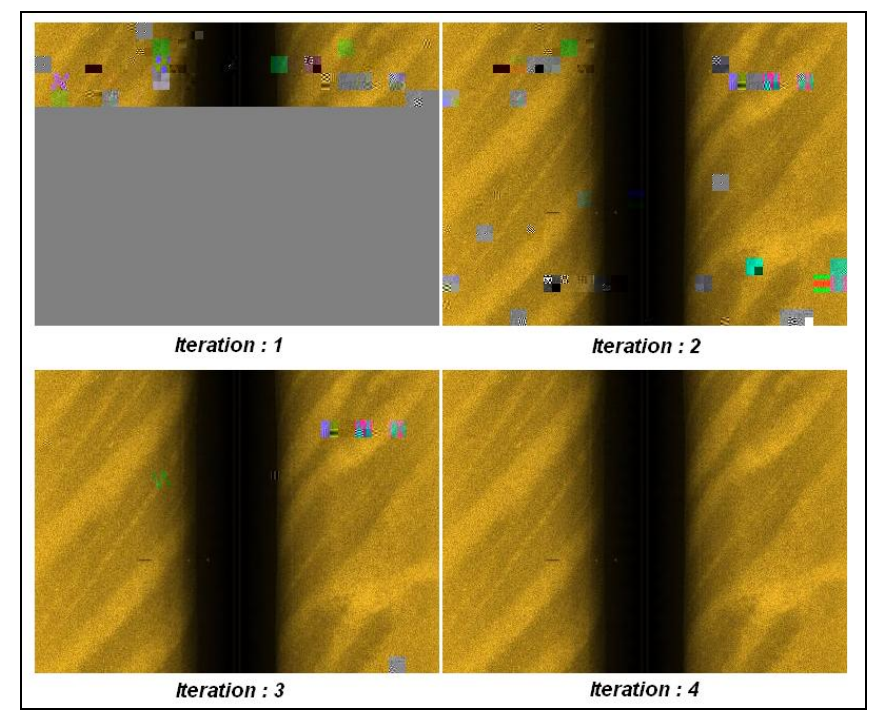

Figure 4. Images after channel decoding (1, 2, 3 and 4 iterations)

\section{B. River trials}

To validate and evaluate TRIDENT system performance over horizontal shallow transmissions, river trials were carried out in November 2009 in Penfeld Brest river. Two carrier frequencies (17.5 or $34.0 \mathrm{kHz})$, and QPSK modulation, were checked during this trials phase, data bit rate in the range 5.8 to $17 \mathrm{kbps}$. Transmitterreceiver distance was about $400 \mathrm{~m}$ and around $7 \mathrm{~m}$ depth. The receiver was on board a boat while the emitter was $5 \mathrm{~m}$ depth below a buoy. In reception, acoustic signals were 
received on 4 sensors, interspaced close to $20 \mathrm{~cm}$ (around 5 wavelengths). First hydrophone was 5 meters depth (about 1 meter below the ship's draught).

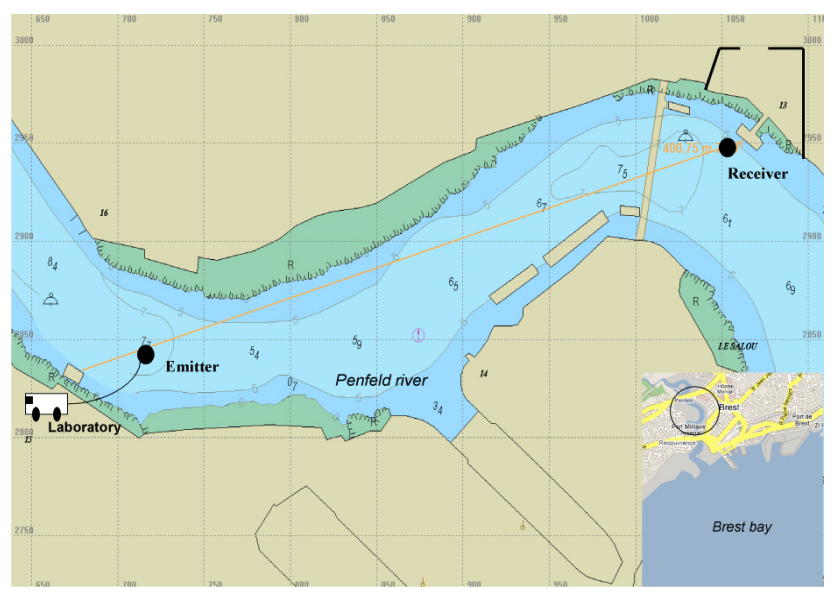

Figure 5. Sea trial site

Sea trials began by the transmission of a speech signal protected by a RS BTC. Data were emitted for 150 seconds at $11.2 \mathrm{kbps}$ with QPSK modulation over a $17.5 \mathrm{kHz}$ carrier frequency.
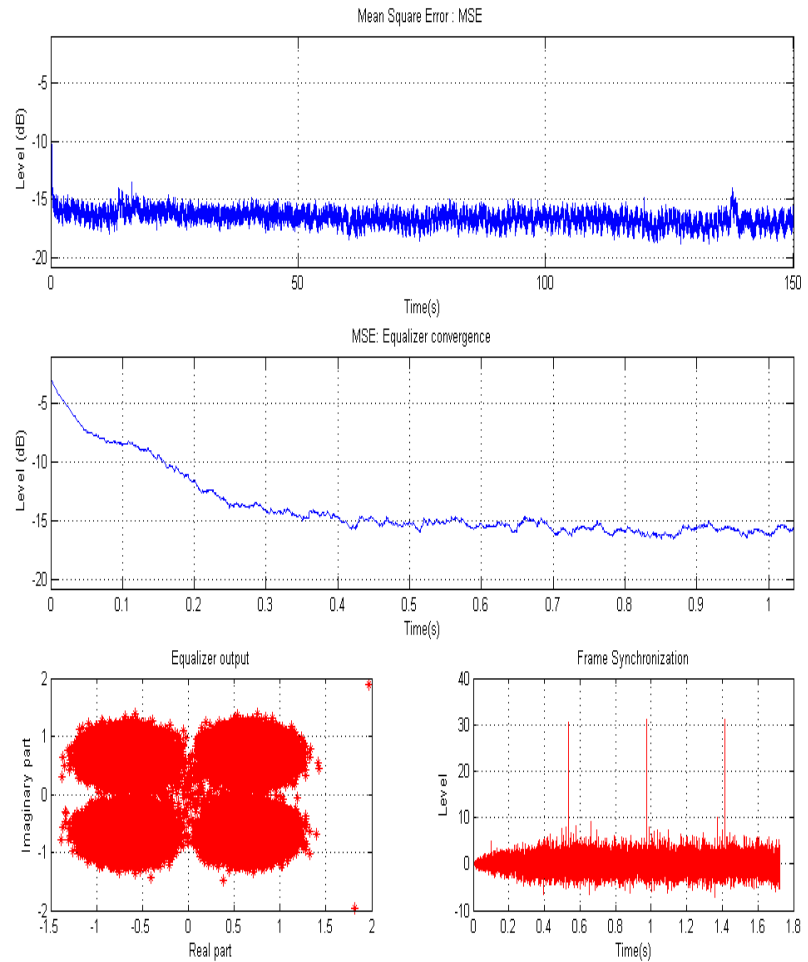

Figure 6. MSE for $150 \mathrm{~s}$ of signal, output equalizer constellation
Both equalizer output constellation and Mean Square Error (MSE) can be seen in Figure 6. Output constellation and MSE also showed the success of the equalization process. A very good overall behavior is to be noted during this sequence. It clearly appears that the transmission was processed without any kind of difficulties. In this trial, phase ambiguity was solved using a DC LLR. After both phase ambiguity correction and decoder synchronization, the BER at the decoder input $\left(\mathrm{BER}_{\text {in }}\right)$ was $1.010^{-4}$ and, no error remained at the output. For a number of 342 frames transmitted, 115 were detected false (one or more errors) at the channel decoder input. At the output, all frames were free of error. Equalizer converging time was only $0.13 \mathrm{~s}$. During the convergence stage, frames may be lost (only a very small lot of them during this trial)

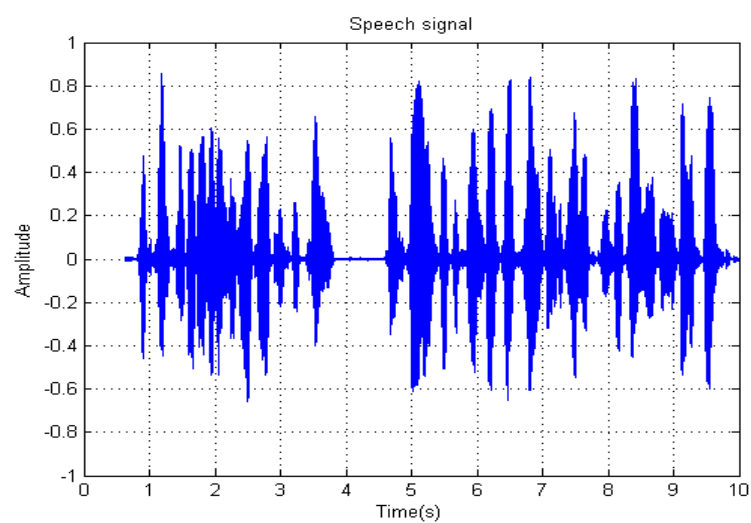

Figure 7. Speech signal

Figure 7 shows 10 seconds of the transmitted signal during this trial. The speech signal was correctly decoded. The transmission bit rate was $11.6 \mathrm{kbps}$ with $2.4 \mathrm{kbps}$ used for the speech signal. It is possible to use the remaining bit rate for other information (text, image).

All the 100 experiments carried out during these trials concluded successfully, giving also very good results.

\section{CONCLUSION AND PERSPECTIVES}

This paper presents the extension of the TRIDENT system, developed by GESMA and TELECOM Bretagne. This underwater acoustic link is able to transmit different kinds of data as text, images and speech. A blind spatiotemporal equalizer is used to reduce different shallow underwater acoustic effects. To improve the underwater acoustic link performances and obtain a higher code rate, Reed Solomon Block Turbo Codes (RS BTC) was tested. The goal was to decrease the BER from $10^{-2}$ to $10^{-4}$. A 
differential coding has been used to solve the phase ambiguities.

First sea trials show that real time transmission of speech and images were carried out in the underwater acoustic channel in stationary state on 400 meters range. One hundred experiments were then carried out on this site (Brest river). All configurations were successful tested for different bit rate with frequencies (17.5 and $34 \mathrm{kHz})$.

Next sea trials will be carried out by end of march in the bay of Brest. These sea trials will check the channel coding in real conditions.

\section{REFERENCES}

[1] J. Trubuil, G. Lapierre, T. Gall, J. Labat, 'Real-time high data rate acoustic link based on spatio temporal blind equalization: the TRIDENT acoustic system', Proc. OCEANS 2002, Biloxi, Vol.4, pp2438-2443.

[2] J. Labat and C. Laot, 'Blind adaptive multiple input decision feedback equalizer with a self optimized configuration', IEEE trans on Comm, Vol. 49, $\mathrm{N}^{\circ} 4$, April 2001.

[3] A. Goalic, J. Trubuil, N. Beuzelin, 'Channel coding for underwater acoustic communication system', Oceans 2006, September 18-21, Boston, Ma, USA.

[4] J. Trubuil, A. Goalic and N. Beuzelin, 'Synchronization and channel coding in shallow water acoustic communication', Oceans 2008, September 15-18, Québec, Canada.

[5] F. J. Macwilliams and N.J.A. Sloane, "The theory of error correcting codes", North-Holland publishing company, 1978, pp. 567-580.

[6] E. R. Berlekamp, "Algebraic coding Theory", McGraw-Hill Book Company, New York, 1968

[7] D. Chase, "A class of algorithms for decoding block codes with channel measurement information", IEEE trans. Inform. Theory, vol IT-8, Jan. 1972.

[8] R, Pyndiah, A, Glavieux, A, Picart and S. Jacq, "Near optimum decoding of product codes", in Proc. Of IEEE Globecom '94, vol. 1/3, Nov.-Dec. 1994, pp.339-343 